\title{
Translingüismo: revisión de la literatura y aplicación didáctica para la enseñanza de ELE y ELE2
}

ANA M. PINO RODRÍGUEZ

Facultad de Educación, Economía y Tecnología del Campus de Ceuta. Universidad de Granada

apino@ugr.es

FERNANDO TRUJILLO SÁEZ

Facultad de Educación, Economía y Tecnología del Campus de Ceuta. Universidad de Granada

ftsaez@ugr.es

ANTONIO GONZÁlEZ VÁZQUEZ

Facultad de Educación, Economía y Tecnología del Campus de Ceuta. Universidad de Granada

agv@ugr.es

Resumen: El translanguaging o translingüismo supone el uso flexible de la totalidad de los recursos lingüísticos a disposición de los usuarios de más de una lengua. Ha cobrado relevancia a lo largo de los últimos años, y lo ha hecho, especialmente, en relación al tratamiento del multilingüismo en el ámbito educativo. Está aún en expansión y, por ello, es posible encontrar definiciones diversas del mismo, lo cual hace del translanguaging una voz polisémica cuyas acepciones pueden interpretarse en distintos planos: el cognitivo, el discursivo y el sociopolítico. Cualquiera de ellas tiene implicaciones concretas a pie de aula y todas determinan un giro en los estudios sociolingüísticos actuales. Este giro queda reflejado en la actualización del Marco Común Europeo para las Lenguas (2018) y viene a trasladar el foco de atención de la investigación académica y de la Didáctica de Lenguas extrajeras desde las propias lenguas, como entidades discretas, hasta sus usuarios.

Palabras clave: Translingüismo, enseñanza de lenguas, usuarios lingüísticos.

Translanguaging: Literature Review and Didactic Uses in Teaching Spanish as a Foreign and Second Language

Abstract: Translanguaging implies the flexible usage that language users make of the whole amount of linguistic resources at their disposal. It has gained relevance along the last years, especially in relation to the treatment of multilinguism in educational contexts. It is still being developed, what explains that it is posible to find different definitions of translanguaging. This makes it a polysemic word whose various meanings can be interpreted on different levels: cognitive, discursive and sociopolitical. Any of these interpretations has practical implications in class and all of them influence a turn in current sociological studies. This turn is reflected upon the updated version of the Common European Framework of Reference for Languages (2018) and transfers the attention focus of academic research and Foreign Language Teaching from languages, as discrete units, to their users.

Keywords: Translanguaging, language teaching, language users. 


\section{Introducción}

Habiendo sido acuñado en la década de los 80 del siglo XX, el translanguaging translingüismo en español- ha ido cobrando relevancia a lo largo de los últimos años al amparo de conceptos como plurilingüismo, heteroglosia y transmedia y en comparación con otros como code-switching o alternancia de código y code-mixing, traducción y mediación, y lo ha hecho, especialmente, en relación al tratamiento del multilingüismo en el ámbito educativo (Blackledge y Creese, 2014: 3). Constituye, así, un concepto relativamente nuevo y polisémico que está todavía en plena expansión. Por este motivo, a lo largo de este artículo se aclarará el sentido del mismo, acudiendo primero a sus orígenes $\mathrm{y}$, luego, a distintas definiciones que sirven para conceptualizar el translingüismo a través de una interpretación en tres planos: el cognitivo, el discursivo y el socio-político. A continuación, se vincularán translingüismo y mediación a través de las perspectivas complementarias que el Volumen de Acompañamiento del MCER (2018) utiliza para describir a los usuarios/aprendices de lenguas. Finalmente, se darán a conocer posibles fórmulas mediante las cuales llevar el translingüismo al aula.

\section{Claves del translingüismo}

El término galés trawsieithu -del cual procede el vocablo anglosajón translanguaginges acuñado en la década de los 80 por el educador Cen Williams para describir un método de enseñanza bilingüe desarrollado en Gales en los niveles de Educación Secundaria. Concretamente, a través del mismo se propone el uso sistemático del galés y del inglés en una única secuencia didáctica (Lewis, Jones y Baker, 2012). Este método se orienta así a la promoción de aprendizajes a partir de la utilización de dos lenguas en una misma clase, de modo que, por ejemplo, mientras a los estudiantes se les ofrece cierto input lingüístico en un idioma concreto, luego puede pedírseles que generen determinado producto en otro idioma. Por tanto, la tarea se desarrolla en dos lenguas que quedan conectadas a través del significado y la comprensión (Beres, 2005: 104), favoreciendo aprendizajes más significativos y duraderos para los y las aprendices y permitiendo a los individuos bilingües - bien sean discentes, bien docentes- poner en juego todas aquellas herramientas presentes en su repertorio lingüístico. Este repertorio, en cualquier caso, no debe ser concebido como algo estable y limitado a un enclave geográfico, sino como un recurso fluido, flexible y, por tanto, adaptable a las diferentes circunstancias que van surgiendo según el momento y el lugar dados (Busch, 2014: 22).

En este contexto, el translingüismo, más allá de la mera traducción, se convierte en una técnica pedagógica empleada en las aulas bilingües para conseguir que los procesos de enseñanza y aprendizaje sean más efectivos y que las lenguas adicionales incorporadas al aula sean más comprensibles (García, 2009: 153). Como resultado, el translingüismo puede definirse como «una práctica de enseñanza basada en cambiar, deliberadamente, el idioma del input y del output» (García y Wei, 2014: 64), reflejando así el «proceso por el que los estudiantes bilingües actúan de manera bilingüe en un sinfín de modos multimodales en clase» (ibid., 65).

Ya comenzado el siglo XXI, el concepto de trawsieithu es retomado por otros autores. Es entonces cuando Colin Baker le da la denominación de translanguaging y cuando Ofelia García amplía sus implicaciones al aproximarse a él de forma holística, considerando que el translingüismo promueve un bilingüismo dinámico por el modo en que favorece la 
construcción de significado, la visión del conjunto de lenguas de un hablante como un único repertorio lingüístico, el desarrollo de una conciencia crítica sobre las políticas lingüísticas y las desigualdades que estas acarrean, la construcción de la propia imagen y el cambio de la visión de otredad (García, 2014). Este modo de entender el translingüismo responde a la necesidad de atender al contexto de las nuevas pedagogías bilingües que demandan las tensiones de un mundo globalizado y caracterizado por el flujo continuo tanto de personas migrantes con bagajes diversos, como de informaciones $\mathrm{y}$ comunicaciones que adoptan formatos multimodales y plurilingües.

En tal escenario, las prácticas lingüísticas han de entenderse desde una perspectiva dinámica, puesto que deben ajustarse continuamente a las situaciones comunicativas multimodales y multilingües que se plantean sobre el terreno, de ahí que este bilingüismo dinámico nos remita, por un lado, al plurilingüismo, entendido, grosso modo, como la habilidad de los usuarios de distintas lenguas para utilizar esos idiomas en distinto grado y con diferentes propósitos (MCERL, 2001, 4). Por otro lado, además, viene a reconocer estructuras y prácticas sociales cada vez más complejas que retratan un escenario comunicativo de súper diversidad (Vertock, 2007).

El análisis y la reflexión en torno a esta realidad globalizada y transmediática ha recuperado para el ámbito de investigación sociolingüística la noción de heteroglosia, que pone el foco sobre la complejidad y la movilidad de las sociedades contemporáneas, así como sobre la naturaleza dinámica de la construcción de las identidades lingüísticas y la complejidad social de cada discurso ${ }^{1}$ (Blackledge y Creese, 2014). El translingüismo crece a la luz de esta compleja noción, del mismo modo que lo han hecho todo un conjunto de términos como el bilingüismo flexible -flexible bilingualism (Creese y Blackledge, 2010)-, code-meshing (Canagarajah, 2011), polylingual languaging (Jørgensen, 2010), las variedades urbanas emergentes - contemporary urban vernaculars (Rampton, 2011), el metrolingualismo -metrolingualism (Otsuji y Pennycook, 2011) - o la práctica translingual -translingual practice (Canagarajah, 2013)-(Blackledge y Creese, 2014: 2). A pesar de las diferencias más o menos sutiles que los puedan separar, todos ellos comparten una concepción de las lenguas como recursos sociales cuyos usos carecen de márgenes claramente establecidos, de modo que los procesos de generación de significados vinculados a dichos usos quedan abiertos a la incorporación de signos procedentes de los repertorios comunicativos de cada individuo.

Precisamente, la perspectiva común compartida por esos términos lleva a situarlos en relación al plurilingüismo del mismo modo en que se hace desde el Marco Común Europeo de Referencia para las Lenguas, según el cual «[a] host of similar expressions now exist, but all are encompassed in the term plurilingualism» (CEFR Companion Volumen, 2018, p. 28). Con respecto al mismo, el MCER ya decía en 2001 que:

el enfoque plurilingüe enfatiza el hecho de que conforme se expande la experiencia lingüística de un individuo en los entornos culturales de una lengua, desde el lenguaje familiar hasta el de la sociedad en general, y después hasta las lenguas de otros pueblos (ya sean aprendidas en la escuela o en la universidad, o por experiencia directa), el individuo no guarda estas lenguas y culturas en compartimentos mentales estrictamente separados, sino que desarrolla una competencia comunicativa a la que contribuyen todos los conocimientos y las experiencias lingüísticas y en la que las lenguas se relacionan entre sí e interactúan. En situaciones distintas, una persona puede recurrir con flexibilidad a partes diferentes de esta competencia [...] (MCER, 2001: 4).

\footnotetext{
${ }^{1}$ En el original, se emplea la palabra voice, que aquí se ha traducido como discurso, en el sentido de práctica semiótica individual.
} 
En esta línea, el Volumen de Acompañamiento del MCER incorpora ciertas novedades (Figura 1), siendo una de ellas dotar a la mediación de una importancia de la que carece en la versión original, de 2001, y que es necesario reconocer actualmente, dada la relevancia que adquiere en sociedades cada vez más diversas y complejas, tanto lingüística, como culturalmente hablando (CEFR Companion Volumen, 2018: 22). Así, la mediación, considerada tradicionalmente en términos de mediación lingüística (traducción/interpretación), pasa ahora a ser valorada como una importante herramienta que permite compaginar el uso extensivo de la lengua meta con la utilización de todos los recursos lingüísticos a disposición de los aprendices (Trujillo Sáez, 2019). Y es que, efectivamente, la mediación resulta «una actividad fundamental en contextos multilingües y un proceso absolutamente natural para usuarios plurilingües» (ibid.). De este modo, mediación y translingüismo van de la mano, ya que la primera puede emplearse como una herramienta didáctica a través de la cual desarrollar procesos translingüísticos de construcción de significado en el aula. O, visto de otra manera, el translingüismo constituye un enfoque pedagógico que valora, incluye e incorpora los repertorios completos de las y los estudiantes, así como sus propias visiones del mundo ${ }^{2}$ (Blackledge y Creese, 2014: 13) y se vale de la mediación para conseguirlo, «making meaning, sharing experiences, finding understandings, and knowledge through the use of two languages» (Baker, 2011: 288).
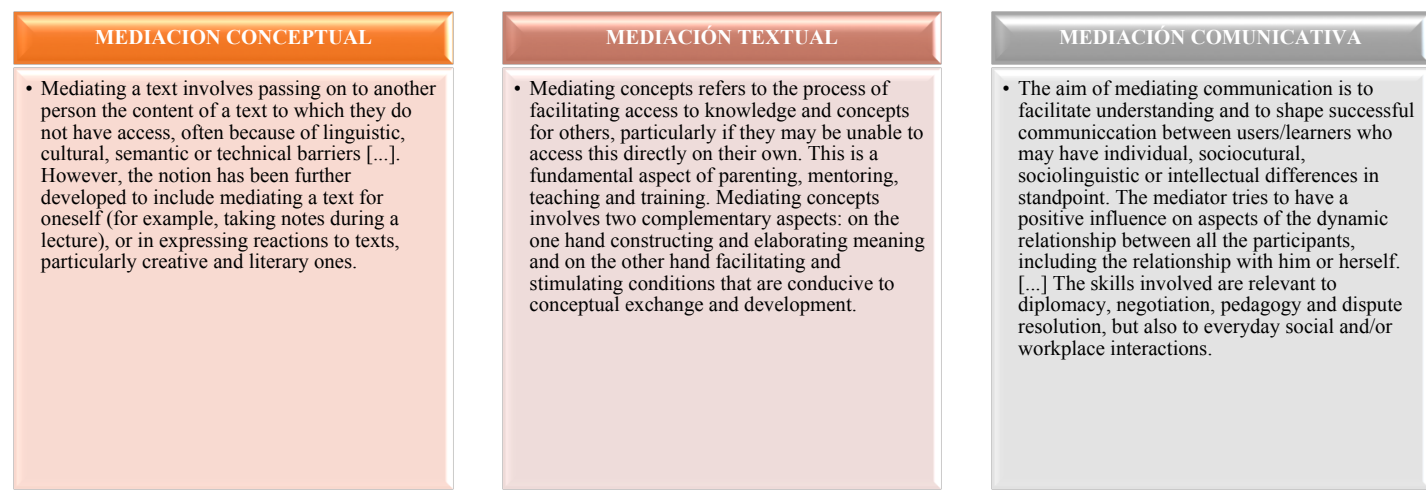

Figura 1. Tipos de mediación incluidos en el CEFR Companion Volume (2018, 106-107)

Así las cosas, el translingüismo trasciende el conjunto de las prácticas y estrategias que utilizan los hablantes bilingües para construir significados y se convierte en un instrumento que da voz al multilingüismo y a la multiculturalidad, resultando en la manifestación de procesos de negociación y construcción de la identidad que van más allá del mero reflejo de las competencias comunicativas individuales (Hidalgo del Rosario, 2018). Por todo ello, Ofelia García habla del carácter transformativo del translingüismo $\mathrm{y}$ lo describe como «a new languaging reality, a new way of being, acting, and languaging in a different social, cultural, and political context, allowing fluid discourses to flow and giving voice to new social realities» (García, 2014: 11-12) -refiriéndose la acción de languaging al desarrollo de una idea, «talking the idea through and hence articulating the throughts» (CEFR Companion Volume, 2018: 33)-.

\footnotetext{
${ }^{2}$ Cita textual de traducción propia.
} 
Considerando las explicaciones previas, el translingüismo queda configurado como una nueva forma de enfocar el plurilingüismo, al centrar su mirada no sobre las lenguas, sino en las personas, en sus prácticas lingüísticas y en sus modos de dar sentido al mundo que les rodea, teniendo ello consecuencias sobre las dinámicas que se generan en las aulas, dentro de las cuales «[s]eeing learners as plurilingual, pluricultural beings means allowing them to use all their linguistic resources when necessary, encouraging them to see similarities and regularities as well as differences between languages and cultures» (CEFR Companion Volumen, 2018, p. 27).

\section{Translingüismo: definiciones e interpretaciones complementarias}

Distintas aproximaciones al término translingüismo han dado lugar a una profusa lista de definiciones, algunas de las cuales ya han sido referidas en los párrafos anteriores. Las mismas lo caracterizan, de manera general, como el conjunto de actuaciones que las personas bilingües realizan para maximizar su potencial comunicativo (García, 2009: 140), pero también es posible encontrar otras acepciones que van desde planos muy concretos y particulares - «an action undertaken by pluriligual persons, where more than one language may be involved» (CEFR Companion Volumen, 2018: 28)- hasta otros más amplios que lo valoran como la herramienta comunicativa más importante con la que podemos contar en el escenario mediático actual (García, 2009: 140), dentro del cual, como ya sabemos, la comunicación lingüística trasciende las lenguas individuales y los mensajes basados en el uso de un solo código. Considerando todo este abanico de opciones, podemos pensar que el translingüismo está asociado a:

- la creatividad, ya que implica la expansión del repertorio lingüístico de cada hablante;

- la práctica interna y la negociación de la propia identidad a través de la integración de los recursos lingüísticos;

- el pensamiento crítico, puesto que es una forma de fomentar la concienciación social a través de la ruptura de las convenciones lingüísticas y sociales;

- un bilingüismo dinámico, que inspira prácticas pedagógicas centradas en «cambiar, deliberadamente, el idioma del input y del output» (García y Wei, 2014: 64);

- el planteamiento de diseños curriculares que den cabida a la riqueza lingüística de sus destinatarios, a conceptos sociales y a la alternancia de códigos entendida más allá de la mera traducción, del code-switching o de la diglosia.

Para tener una idea clara acerca de todas estas implicaciones, Trujillo Sáez (2019) establece una triple interpretación del término: una cognitiva, otra lingüística y otra discursiva (Figura 2). Así, desde un punto de vista cognitivo, el translingüismo incorpora el conjunto de procesos mentales desarrollados durante aquellas situaciones comunicativas en las cuales distintas lenguas son empleadas de forma dinámica $\mathrm{y}$ funcional (Lewis, Jones y Collin, 2012: 641-654), lo cual ha de explotarse en entornos pedagógicos para dar lugar al desarrollo de la comprensión metalingüística y la conciencia meta-cognitiva de las y los aprendices de lenguas. Mientras, la interpretación discursiva nos conduce a la identificación de aquellas prácticas que las personas bilingües o multilingües emplean para dar sentido al mundo que los rodea (García, 2009). Así, lo que concierne al translingüismo -tanto en su interpretación cognitiva, como en la discursiva-, es la posibilidad de generar una comunicación efectiva donde función está por encima de forma y en la cual se combinen acción cognitiva y producción lingüística 
(Lewis, Jones y Baker, 2012: 641-654). Como resultado, el translingüismo es una práctica muy significativa, «pedagogically to teach, but also cognitively to learn» (García, 2009: 156).

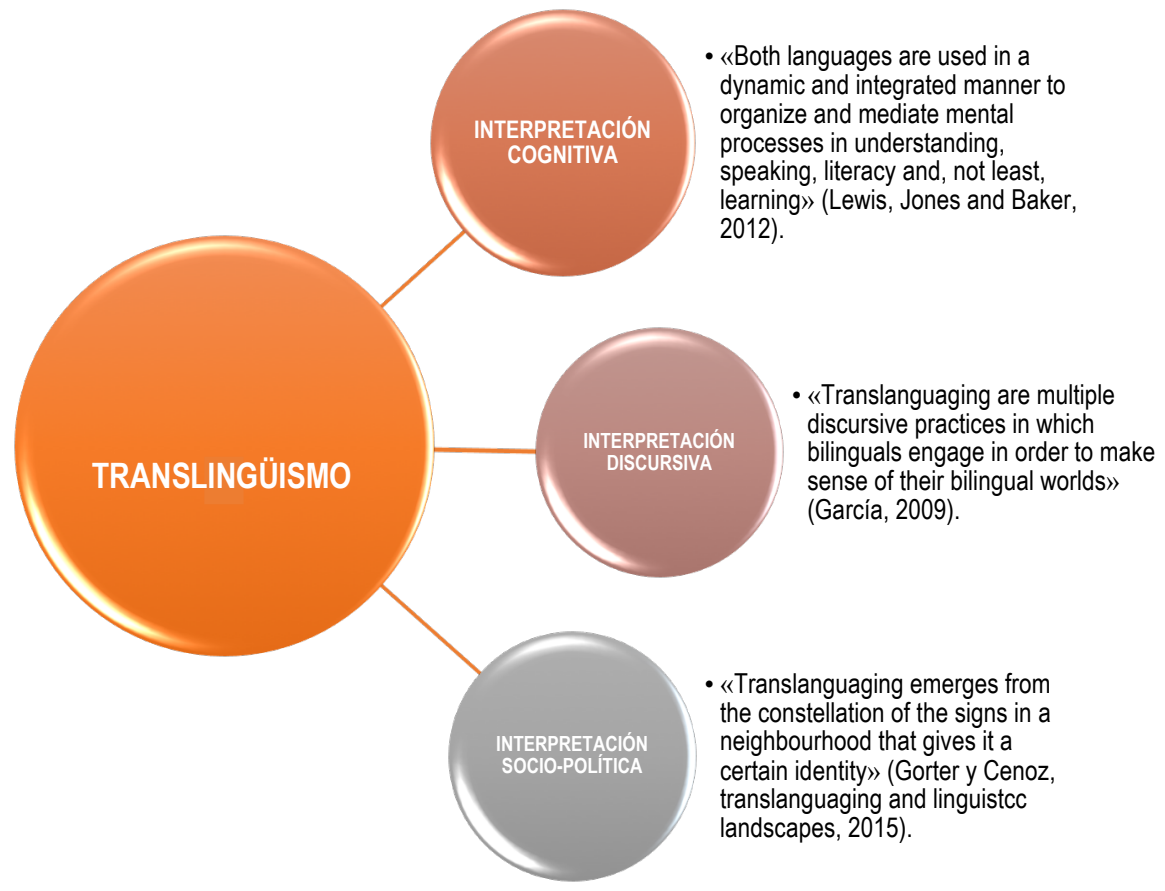

Figura 2. Interpretaciones del translingüismo, según Trujillo Sáez (2019)

Más que centrarse en las lenguas de los hablantes, el translingüismo se ocupa de sus prácticas y de las estrategias que estos emplean «para construir significados y dar voz al multilingüismo y multiculturalidad» (Hidalgo del Rosario, 2018: 136). Así, ya en el plano socio-lingüístico, el translingüismo da lugar a una reivindicación de la propia identidad mediante el reconocimiento de la multiplicidad de voces contenidas en los discursos y de su posición en un continuo dinámico y multilingüe que no parte del monolingüismo (García, 2014: 145). En esta línea, Blackledge y Creese (2014) entienden que el translingüismo ayuda a los estudiantes a negociar distintas identidades culturales y lingüísticas en casa y en su comunidad, mientras que Wei puntualiza que el translingüismo promueve la transmisión de información y la representación de valores, identidades y relaciones sociales (Wei, 2011: 1223; cit. por Gorter y Cenoz, 2015).

García y Wei (2011), por su parte, nos ofrecen una descripción de translingüismo que viene a unificar las tres interpretaciones aludidas, al caracterizarlo del siguiente modo: «traslanguaging is a process whereby individuals use various meaning-making signs in order to adapt and actively participate in different societal and linguistic situations critically and with cognitive engagement and creativity» (García y Wei, 2014: 66-68; cit. por Beres, 2015: 110).

\section{Algunas aplicaciones didácticas del translingüismo}

Desde que Baker y García dieran impulso al translingüismo, distintos estudios se han centrado en el concepto para valorar su uso dentro de contextos académicos y sociales 
diversos. Beres $(2015,109-111)$ recoge esos ámbitos de investigación en relación al translingüismo y sitúa los trabajos hallados en torno a: estrategias de escritura (Canagarajah, 2011); prácticas multilingües en escuelas complementarias en Reino Unido (Creese y Blackedge, 2011); escritura y creación de mensajes matemáticos; alfabetizaciones transnacionales basadas en identidades, habilidades y relaciones sociales transfronteriza (Hornberger y Link, 2012); pedagogías dinámicas en escuelas multilingües y multiculturales (García y Sylvan, 2011); languaging (García, 2011, 2014b); espacios sociales y culturales (Wei, 2011); o con los procesos cognitivos ligados al uso de dos lenguas en la misma situación -input-output thinking- (Kano, 2013). Investigaciones más recientes se centran en describir y analizar el uso del translingüismo en comunidad (Birmingham), en explicar cómo el translingüismo y la traducción son empleados por personas de distintas procedencias lingüísticas y culturales para comunicarse con éxito, y en demostrar a través de experimentos neurológicos cuáles son los beneficios de la alternancia entre lenguas de cara a la adquisición de nuevos conocimientos.

A pesar de diversidad de vías, es necesario seguir ampliando y profundizando en la investigación ya en curso para obtener conclusiones generalizables especialmente en el ámbito educativo, de modo que los sujetos implicados en los procesos de enseñanza y aprendizaje conozcan qué elementos relativos del uso pedagógico del translanguaging hay que tomar en consideración para mejorar la competencia lingüística del alumnado (Canagarajah, 2011: 1-28).

García (2009: 148) entiende que para que el translingüismo prospere como herramienta didáctica es necesario abonar el terreno educativo con grupos constituidos por alumnado de perfiles lingüísticos diversos y enfoques educativos centrados en la individualización, de modo que se tengan en cuenta tanto las características singulares de cada estudiante, como su interacción colaborativa. Desde el Volumen de Acompañamiento del MCER (2018: 27) se destaca el Enfoque Orientado a la Acción, que implica el desarrollo de tareas también colaborativas, como una opción metodológica adecuada cuando el acento no se pone sobre la lengua sino sobre la generación de productos lingüísticos que respondan a un propósito real (por ejemplo, una salida planificada, la elaboración de un póster o de un blog, la organización de un festival, la elección de una candidatura, etc.) (CEFR Companion Volume, 2018: 27).

Trujillo Sáez (2019) apuesta por emplear mediación y translingüismo en las aulas con el objetivo de responder a las demandas del panorama comunicativo presente y futuro y hace un repaso por enfoques, estrategias y recursos metodológicos que los centros educativos pueden utilizar para alcanzar ese objetivo. Así, este autor hace también referencia al Enfoque Orientado a la Acción, para seguir el Enfoque de Experiencia lingüística y la implementación de proyectos de interpretación cultural; los Textos identitarios, de Jim Cummins y Early (2010); el movimiento hacia fuera del aula, basado en la promoción de una socialización rica a través del Aprendizaje cooperativo; el Aprendizaje entre Iguales; las distintas actuaciones enmarcadas dentro del concepto de aprenseñar (Durán Gisbert, 2014) o los hablantes invitados (guest speakers, McNett, 2001; Murray, 1998; Varvel, 2001; cit. por Hemphill y Hemphill, 2006)-y el movimiento hacia dentro del aula -mediante la presencia de agentes externos, el diseño de proyectos multimedia y multilingües, los paisajes lingüísticos (Gorter y Cenoz Iragui, 2013; González Vázquez y Trujillo Sáez, 2018) o el estudiante investigador (Norton, 2013)-; 
los momentos de comentario metalingüístico de Palmer, Martínez, Mateus y Henderson $\mathrm{y}$ las actividades de conciencia metalingüística; el translingüismo planificado y espontáneo (code-switching y traducción) y el centro educativo como entorno ecológico multilingüe.

\section{Conclusiones}

Como se ha visto, el translingüismo es un concepto complejo y en expansión que tiene que ver con el uso flexible de los repertorios lingüísticos de las personas bilingües, motivo por el cual se ha vinculado en ocasiones a otros muchos conceptos emergentes y especialmente a las nuevas pedagogías bilingües y a una teoría sociolingüística crítica. Se ha comparado a la traducción y a la alternancia de código, aunque el translingüismo supera y a la vez incluye estas nociones, al entenderse que el hablante multilingüe posee un único repertorio lingüístico, conformado por distintos elementos lingüísticos y semióticos, de los cuales utiliza aquellos más adecuados al contexto sociocultural en que se halle. Así, el translingüismo refleja la identidad singular de cada individuo, el valor heteroglósico de su discurso y el contexto ecológico al que se vincula.

El interés que ha despertado entre la comunidad científica parece despejar dudas respecto a su valor en el terreno educativo, pero lo cierto es que, a pesar de las muchas opciones metodológicas a las cuales podemos vincular su uso en el ámbito educativo, es necesario continuar profundizando en él para conocer con precisión su verdadero potencial pedagógico y sus posibilidades en contextos distintos, dentro y fuera del aula de lengua extranjera, con alumnado diverso y vinculado al uso de herramientas tanto digitales, como analógicas. Desde luego, el conocimiento preciso sobre sus componentes y la configuración y diseño de pautas docentes que se valgan del translingüismo para mejorar las competencias plurilingües e interculturales de su alumnado pueden convertir este concepto en un activo de aprendizaje de gran valor educativo en las aulas del siglo XXI.

\section{Bibliografía}

BERES, A. M. (2015). "An overview of translanguaging: 20 years of "giving voice to those who do not speak"», Translation and Translanguaging in Multilingual Contexts, (1)1, 103-118.

Blackledge, A. y Creese, A. (2014). «Heteroglossia as Practice and Pedagogy». En A. Blackledge y A. Creese (eds.); Heteroglossia as Practice and Pedagogy (pp. 1-20). Springer: Dordrecht.

Busch, B. (2014). «Building on Heteroglossia and Heterogeneity: The Experience of a Multilingual Classroom». En A. Blackledge y A. Creese (eds.); Heteroglossia as Practice and Pedagogy (pp. 21-40). Springer: Dordrecht.

CANAgARAJAH, S. (2011). Translanguaging in the classroom. Emerging issues for researcha and pedagogy, Applied Linguistics Review, 2, 1-28.

Colin, B. (2011). Foundations of Bilingual Education and Bilingualism. Bristol: Multilingual Matters.

CONSEJO DE EUROPA (2002). Marco común europeo de referencia para las lenguas: aprendizaje, enseñanza, evaluación. Madrid: Ministerio de Educación, Instituto Cervantes y Anaya. 
CONSEJO DE EuROPA (2018). Common European Framework for Languages. Companion Volumen with new descriptors. Recuperado el 22/05/19, de: https://rm.coe.int/cefrcompanion-volume-with-new-descriptors-2018/1680787989

CUMmins, J. Y EARLy, M. (2010). Identity Texts: The Collaborative Creation of Power in Multilingual Schools. Stokeon-Trent, Reino Unido: Trentham Books.

DURÁN GISBERT, D. (2014). Aprenseñar: evidencias e implicaciones educativas de aprender enseñando. Madrid: Narcea.

GARCíA, O. (2009). Education, «Multilingualism and Translanguaging in the 21st Century». En T. Skutnabb-Kangas, R. Phillipson, A. K. Mohant y M. Panda; Social Justice in Multilingual Education (pp. 140-158). Bristol: Multilingual Matters.

GarcíA, O., Y SYlvan, C. E. (2011). «Pedagogies and practices in multilingual classrooms: Singularities in pluralities». The Modern Language Journal, 95(3), 385-400.

GARCÍA, O. y WEI, L. (2014). «Translanguaging and Education». En Translanguaging: Language, Bilingualism and Education (pp. 63-77). London: Palgrave MacMillan.

GorTer, D. y CenOz IRAGUi, J. (2015). «Translanguaging and linguistic landscape», Linguistic landscape, 1, 1/2, 54-74.

HIDALGO DEL RosARIO, A. V. (2018). «Implicaciones lingüísticas, sociales y pedagógicas del translenguaje frente a la alternancia de código», Revista de la Asociación Europea de Profesores de Español. El español por el mundo, 1, 113-122. Recuperado el 24/05/2019, de https://cvc.cervantes.es/ensenanza/biblioteca_ele/aepe/revista_01_01_2018.htm

HEMPHILl, L. S. Y HEMPHILL, H. H. (2007). «Evaluating the impact of guest speaker postings in online discussions», British Journal of Educational Technology, (38)2, 287 293, DOI: $10.1111 / \mathrm{j} .1467-8535.2006 .00622 . \mathrm{x}$

JøRGENSEN, J. N. (2010). Languaging: Nine years of poly-lingual development of young Turkish-Danish grade school students. Copenhagen Studies in Bilingualism. Copenhagen, Dinamarca: Facultad de Humanidades de Copenhague. Recuperado el 30/06/2019, de https://andetsprogsforskning.ku.dk/forskning/koebenhavnerstudier_i tosprogethed_/manuskripter/Bind_K15_-_J._N._J_rgensen_-_Languaging_-_Vol._1.pdf

KANO, N. (2012). Translanguaging as a process and a pedagogical tool for Japanese students in an English writing course in New York. Tesis doctoral. Teachers College: Columbia University, Nueva York (Estados Unidos).

LEWIS, G, JONES, B. y BAKER, C. (2012). «Translanguaging: origins and development from school to street and beyond», Educational Research and Evaluation, 18, 7, 641-654, DOI: $10.1080 / 13803611.2012 .718488$.

LEWIS, G., JONES, B., Y BAKER, C. (2012). «Translanguaging: Developing its conceptualisation and contextualisation», Educational Research and Evaluation, 18, 7, 655-670.

MCNetT, M. (2001). «Virtual guest lecturers: bring a world of experts to your students». Sixth Annual Teaching in the Community Colleges Online Conference. Recuperado el 11/09/2005, de: http://makahiki.kcc.hawaii.edu/tcc/tcon01/papers/monett.html

MURRAY, D. E. (1988). «Computer-mediated communication: implications for ESP [English for Specific Purposes]», English for Specific Purposes, 7, 3-18. 
NORTON, B. (2013). Identity and Language Learning. Extending the conversation. Bristol, Buffalo, Toronto: Multilingual Matters.

Otsuj, E., y PENNYCOOK, A. (2011). «Social inclusion and metrolingual practices», International Journal of Bilingual Education and Bilingualism, 14, 413-426.

Palmer, D. K., Mateus, S. G., Martínez, R. A. y Henderson, K. (2014). «Reframing the debate on language separation: Toward a vision for translanguaging pedagogies in the dual language classroom», The Modern Language Journal, 98, 3, 757-772, DOI: $10.1111 / \bmod 1.12121$.

RAMPTON, B. (2011). «From "multi-ethnic adolescent heteroglossia" to "contemporary urban vernaculars"», Language \& Communication, 31, 276-294.

TRUjILlo SÁEZ, F. (2019). «Mediación y translingüismo en el aula de idiomas. Propuestas teóricas y prácticas». Recuperado el 14/05/19, de: https://es.slideshare.net/ftsaez/mediacin-y-translingismo-en-el-aula-de-idiomas132938409

TrujILlo SÁEZ, F. (2018). Activos de aprendizaje. Utopías educativas en construcción. Madrid: SM.

VARVEL, V. E., JR. (2001). «Guest lecturers in the online environment» (parte 2. a de 2). Pointers \& Clickers. Recuperado el 02/09/2005, de http://www.ion.uillinois.edu/resources/pointersclickers/2001_09/index.asp

VÁZQUEZ, A. G., y SÁEZ, F. T. (2007). «El lugar ideológico de la enseñanza de las lenguas: cuestiones sobre el fenómeno migratorio». Textos de Didáctica de la Lengua y la Literatura, 45, 46-59.

VÁzQUez, A. G., y SÁEZ, F. T. (2018). «Paisajes lingüísticos y translingüismo como propuesta lingüística a una pedagogía de frontera», Cuadernos de Pedagogía, 492, Barcelona: Wolters Kluwer.

Vertovec, S. (2007). «Super-diversity and its implications», Ethnic and Racial Studies, 30, 6, 1024-1054, DOI: 10.1080/01419870701599465

WEI, L. (2011). «Moment analysis and translanguaging space: Discoursive construction of identities by multilingual Chinese youth in Britain», Journal of Pragmatics, 43, 5, $1222-1235$. 